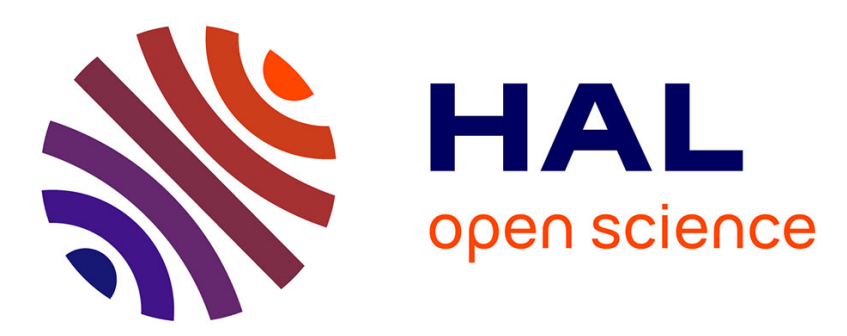

\title{
An extensive 3D dislocation dynamics investigation of stage-I fatigue crack propagation
}

\author{
C. Déprés, G.V. Prasad Reddy, C. Robertson, M. Fivel
}

\section{To cite this version:}

C. Déprés, G.V. Prasad Reddy, C. Robertson, M. Fivel. An extensive 3D dislocation dynamics investigation of stage-I fatigue crack propagation. Philosophical Magazine, 2014, 94 (36), pp.41154137. 10.1080/14786435.2014.978830 . hal-01122452

\section{HAL Id: hal-01122452 \\ https://hal.science/hal-01122452}

Submitted on 11 Jun 2019

HAL is a multi-disciplinary open access archive for the deposit and dissemination of scientific research documents, whether they are published or not. The documents may come from teaching and research institutions in France or abroad, or from public or private research centers.
L'archive ouverte pluridisciplinaire HAL, est destinée au dépôt et à la diffusion de documents scientifiques de niveau recherche, publiés ou non, émanant des établissements d'enseignement et de recherche français ou étrangers, des laboratoires publics ou privés. 


\title{
An extensive 3D dislocation dynamics investigation of stage-I fatigue crack propagation
}

\author{
C. Déprés ${ }^{\mathrm{a} *}$, G.V. Prasad Reddy ${ }^{\mathrm{a}, \mathrm{b}}$, C. Robertson ${ }^{\mathrm{c}}$ and M. Fivel ${ }^{\mathrm{d}}$ \\ ${ }^{a}$ SYMME, Université Savoie Mont Blanc, F-74000 Annecy, France; ${ }^{b}$ Mechanical Metallurgy \\ Division, Indira Gandhi Center for Atomic Research, Kalpakkam, 603102 Tamil Nadu, India; \\ ${ }^{c}$ Service de Recherche de Métallurgie Appliquée, CEA Saclay, 91191 Gif-sur-Yvette, Paris, \\ France; ${ }^{d}$ SIMAP-GPM2, Université Grenoble Alpes-CNRS, F-38000 Grenoble, France
}

\begin{abstract}
Stage-I fatigue crack propagation is investigated using 3D discrete dislocation dynamics (DD) simulations. Slip-based propagation mechanisms and the role of the pre-existing slip band on the crack path are emphasized. Stage-I crack growth is found to be compatible with successive decohesion of the persistent slip band/matrix interface rather than a mere effect of plastic irreversibility. Corresponding crack tip slip displacement magnitude and the associated crack growth rate are evaluated quantitatively at various tip distances from the grain boundary. This shows that grain boundaries systematically amplify slip dispersion ahead of the crack tip and consequently, slow down the stage-I crack growth rate. The results help in developing an original crack propagation model, accounting for the boundary effects relevant to polycrystals. The crack growth trend is then evaluated from calculations of the energy changes due to crack length increments. It is shown that the crack necessarily propagates by increments smaller than $10 \mathrm{~nm}$.
\end{abstract}

Keywords: dislocation dynamics; crack growth; fatigue

\section{Introduction}

Cyclic slip localization and persistent slip bands (PSBs) formation in 316L stainless steel have been extensively modelled and characterized, especially in the low-strain (or high cycle) regime [1-3]. In particular, careful examinations of persistent slip markings during stage-I have shown that fatigue crack initiations mainly occur at the PSB-matrix interface [3-5], or within the PSB itself [5,6]. Initiated cracks propagate essentially under shear mode (mode-II) and along a given crystallographic slip plane. The crack propagation rate is strongly dependent on the dislocation microstructure at the crack tip $[7,8]$, and hence on the crack tip slip displacement (CTSD). From the Bilby, Cottrell and Swinden (BCS) model, Lardner emphasizes the role played by the surrounding microstructure of the crack, and that the CTSD for stationary and fatigue cracks are markedly different $[9,10]$. Thus, it is important to relate the CTSD to plasticity mechanisms and grain morphology.

\footnotetext{
*Corresponding author. Email: christophe.depres@univ-savoie.fr
} 
Several studies have been carried out so far using dislocation dynamics (DD) modelling with a view to simulate fatigue crack propagation under mode-I and mode-II [9-19]. Navarro and de los Rios [9] have developed a 2D micromechanical model based on the continuous distribution of dislocations. This model successfully describes the influence of grain boundaries on the crack tip plastic displacement as a function of the crack length. Subsequently, many authors have conducted complementary discrete dislocation studies [10-20], for example, to identify threshold conditions for stage-I crack propagation [10], to study the influence of the normal stress on the threshold stress intensity factor for stage-I FCP [13] and to examine the growth of mode-I short cracks in a FCC material, in combination with a cohesive surface in front of the crack tip $[14,15]$. In another approach based on the boundary element method (BEM), the crack is represented by sets of dislocation dipoles and crystal plasticity results from the discrete dislocations (or dipole elements) movements along certain slip planes [16-19]. BEM has been used to simulate short crack propagation through simulated microstructures taken as a statistical distribution of grain geometries and crystallographic orientations in 2D space [16]. Zig-zag paths of a stage-I crack propagating in a grain [17] and crack morphologies in mode-I and mode-II were investigated [17-19]. Previous studies, though mainly $2 \mathrm{D}$, were able to capture important aspects of cyclic plasticity and crack growth of the order of a few Burgers vectors. On the other hand, it is well known that 2D simulations do not reproduce many important features such as line tension, crossslip or more generally, the 3D character of dislocation microstructures. For this reason, 2D simulations cannot accurately describe internal stress field evolutions at the grain scale. Moreover, the dislocation lines in 2D simulations are taken as infinite and parallel edge (or screw) dislocations, in contrast with the actual configuration of fcc grains.

Very few 3D DD simulations adapted to cyclic strain have been carried out so far $[20,21]$. For this and other reasons, there are still many open questions regarding transgranular crack propagation mechanisms in actual polycrystals (crack path geometry, effect of grain boundaries, etc.). The goal in this work is to investigate short crack propagation using DD simulations accounting for the inherent complexity of the $3 \mathrm{D}$ space, including realistic boundary effects (namely, grain boundaries and free surfaces). The simulation results are presented in the form of changes in the dislocation structure, the crack morphology and the stored energy associated with the stage-I crack propagation.

\section{Simulation methods: geometries and material parameters}

All the simulations presented here are performed using the TRIDIS DD code, where the dislocation lines are treated as discrete edge-screw segments (see [23] for more details). The main simulation inputs are the initial microstructure and the external applied loading. Dislocation segments move in the simulated space according to an explicit scheme, where the individual segment velocity is proportional to the local effective stress (applied stress plus the internal stress). Interaction with other dislocations (annihilations, junctions) and cross-slip events are evaluated and updated at each time step. The applied loading is feedback controlled based on the Von Mises equivalent plastic strain evolutions, enforcing constant strain rate conditions. The main simulation output is the plastic strain based on dislocation structure evolutions. In this work, the simulated space corresponds to a single FCC grain. This code has been successfully applied and 
validated in different cases of monotonic loading conditions such as tensile straining [24] or nanoindentation [25]. This same numerical tool was used earlier to perform DD simulations of fatigue in homogeneous 3D grains [20,21,26]. The choice of this specific DD code is based on a rather unique compromise between calculation cost, accuracy/ relevance of the results and numerical stability. Thanks to the previous studies conducted in fatigue of $316 \mathrm{~L}$ stainless steels, the range of the input parameters (such as: time step, discretization length, grain size, strain amplitude and cross-slip activation parameters of $316 \mathrm{~L}$ steel), compatible with both a sufficient accuracy and exploitable results in fatigue, is well identified in TRIDIS code.

In the present study, the considered grain geometry (see Figure 1(a)) is taken as a hexagonal prism with a height to circumscribed diameter ratio of 1.0. Three different depths have been tested for the grain: 20,15 and $10 \mu \mathrm{m}$, denoted by $D g$ in this paper. The studied grain is a surface grain with impenetrable grain boundary faces, except one free surface from which dislocations can emerge and modify the surface shape (by plastic step printing). The simulated box can be viewed as a plastic heterogeneity embedded in an infinite elastic medium. This assumption is fully relevant in the high cycle fatigue regime tested herein, where the number of plastically deformed grains is limited and where the deformed grains are randomly distributed across the whole polycrystal. The loading is a cyclic tension-compression applied close to $\left[\begin{array}{lll}1 & 2 & 3\end{array}\right]$ direction, which induces slip mainly on the $a / 2\left[\begin{array}{lll}1 & 0 & 1\end{array}\right]\left(\begin{array}{lll}1 & 1 & 1\end{array}\right)$ slip system (Schmid factor $\left.\sim 0.5\right)$. For all the fatigue simulations, the plastic strain amplitude is fixed to $\Delta \varepsilon_{p}=10^{-4}$. The initial dislocation configuration consists of a set of pinned segments (at least one source per slip system), all of similar length and randomly positioned in the central zone of the grain (so that the grain boundaries could not inhibit the Frank-Read multiplication mechanism). The initial source configuration has no effect on the morphology of the microstructure after sufficient cumulated plastic deformation (generally one full cycle) [20]. Plastic steps induced at the surface by the dislocation microstructure are computed using the specific post-treatment methodology as explained in [21,22]. Image forces are not included in this work, since their influence on grain-scale plasticity is found to be negligible [21], as demonstrated in Section 3.7 in presence of a crack-like geometrical discontinuity. The material parameters considered for the present simulations correspond to $316 \mathrm{~L}$ stainless steel (see Table 1). Finally, screw segments can change their glide plane through thermally activated cross-slip mechanisms. Cross-slip probability $P$ is proportional to $\exp \left(-\frac{\tau_{\mathrm{III}}-\tau_{\mathrm{dev}}}{k T} V_{\text {act }}\right)$, where $\tau_{\mathrm{dev}}$ is the resolved shear stress in the crossslip system, $\tau_{\text {III }}$ a threshold value, $V_{\text {act }}$ is the activation volume [23] and $\mathrm{T}=293 \mathrm{~K}$.

Typical simulations are conducted in two stages. First, a realistic PSB lying along the primary slip system is generated. Then, crack tip plasticity and CTSD is analysed by introducing a crack along the PSB-matrix interface.

\section{Results and discussion}

\subsection{Surface relief formation and crack initiation}

A first DD simulation is carried out to generate a reference PSB microstructure, as described in details in references [20,21]. The main plasticity mechanisms are briefly recalled hereafter, since they are intimately linked to microcrack initiation and the subsequent crack propagation. As the cycles proceed, plasticity results in the concentration 
(a)
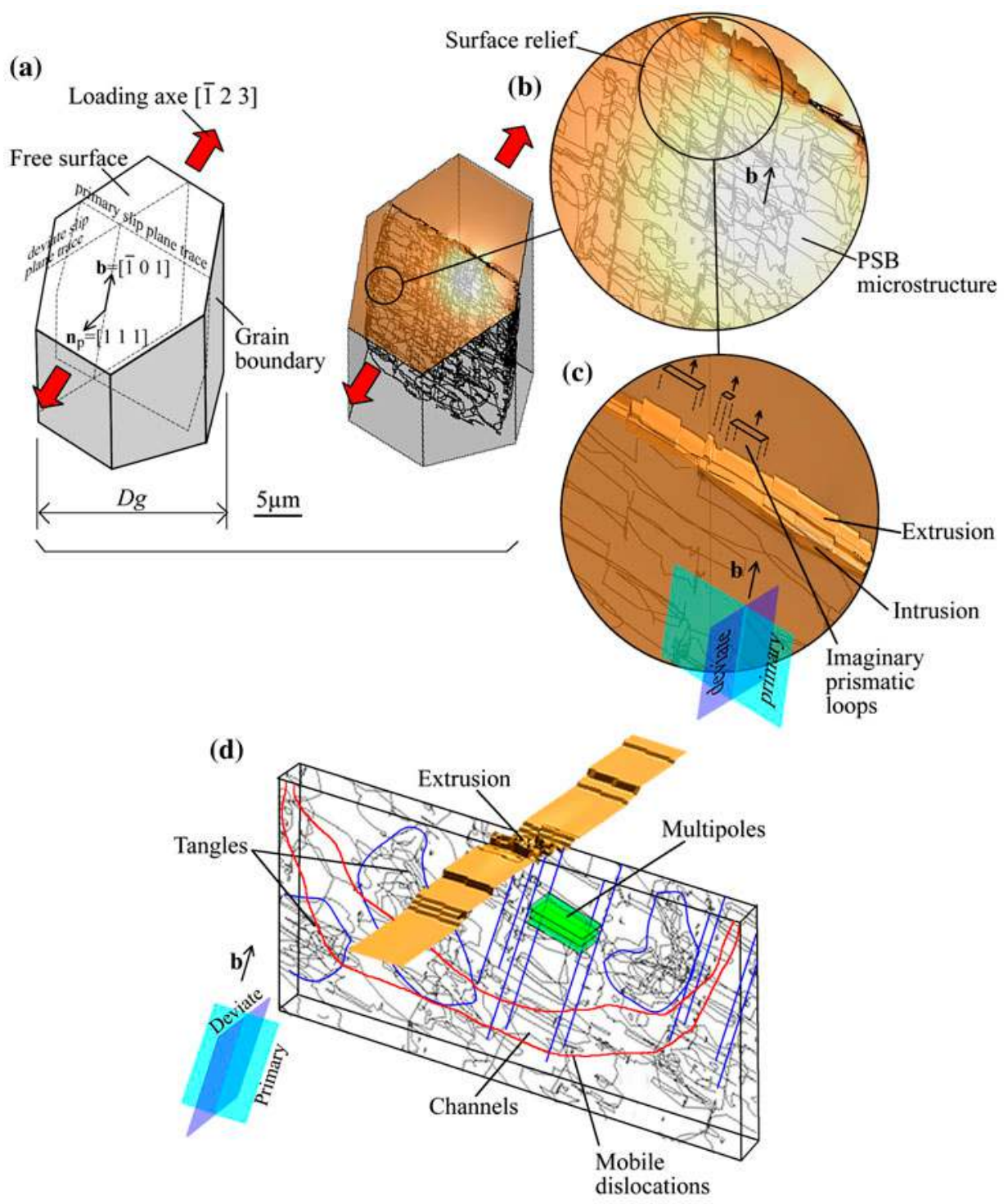

Figure 1. (colour online) Typical fatigue dislocation microstructure developed in 3D DD simulation and associated surface relief (mostly extrusions). The simulations are carried out in a grain oriented for single slip and for plastic strain amplitude $\Delta \varepsilon_{\mathrm{p}}$ in the range $10^{-4}-10^{-3}$. (a) Hexagonal grain including a PSB. (b) Enlarged view of the microstructure showing channels made of prismatic loops and helix structures. (c) Sketch of the virtual dislocation loops (in black colour) emerged out of free surface with subsequent formation of extrusions. (d) PSB microstructure and associated schematic representation [21].

of cyclic slip along the primary slip system $a / 2\left[\begin{array}{lllll}1 & 0 & 1\end{array}\right]\left(\begin{array}{lll}1 & 1 & 1\end{array}\right)$ accompanied by glide plane change on the cross-slip system $a / 2\left[\begin{array}{lll}1 & 0 & 1\end{array}\right]\left(\begin{array}{lll}1 & 1 & 1\end{array}\right)$. After the completion of few cycles, interactions between primary and cross-slipped dislocations lead to the formation 
Table 1. Material parameters (at $300 \mathrm{~K}$ ) and simulation parameters used in the DD simulations.

\begin{tabular}{lccccccc}
\hline $\begin{array}{l}\text { Threshold } \\
\text { stress }\left(\tau_{\text {III }}\right)\end{array}$ & $\begin{array}{c}\text { Viscous Drag } \\
\text { Coefficient, } \\
{\left[\times 10^{-5}\right]}\end{array}$ & $\begin{array}{c}\text { Lattice } \\
\text { friction } \\
\text { stress } \tau^{*}\end{array}$ & $\begin{array}{c}\text { Shear } \\
\text { modulus } \mu\end{array}$ & $\begin{array}{c}\text { Burgers } \\
\text { norm } b\end{array}$ & Time step & $\begin{array}{c}\text { Strain } \\
\text { amplitude } \\
\Delta \varepsilon_{\mathrm{p}}\end{array}$ & $\begin{array}{c}\text { Activation } \\
\text { volume } \\
V_{\text {act }}\end{array}$ \\
\hline $51.2 \mathrm{MPa}$ & $1.06 \mathrm{~Pa} . \mathrm{s}$ & $40 \mathrm{MPa}$ & $81,000 \mathrm{MPa}$ & $2.54 \AA$ & $2 \times 10^{-10} \mathrm{~s}$ & $10^{-4}$ & $1800 b^{3}$ \\
\hline
\end{tabular}

of a typical PSB dislocation structure, as shown in Figure 1. The number of individual PSBs is independent of the grain size for a fixed plastic strain range, as explained in [21]. The grain size, however, affects both the slip band characteristic thickness and the inter-band spacing. Hence, the mean inter-band spacing is proportional to the grain size, whereas the band thickness is proportional to the grain size squared. The PSB microstructure arrangement includes three distinct subarrangements as depicted in Figure 1(d) and [21]. This qualitative description holds true whatever the gain size or applied strain range.

(1) Dislocation multipoles located in channels substructures. These multipoles result from the interaction between primary and cross-slipped dislocations. The multipoles usually rearrange in the form of prismatic loops or helix. The associated surface relief consists of growing extrusions mainly located at the channel/surface intersection (see Figure 1(c)). Note that in the absence of diffusion mechanisms, the prismatic loops and/or helix motion is constrained within the channels.

(2) Tangles surrounding the channels. The tangle zones have been generated at the same time as the multipoles. The local reorganization of the dislocation lines leads to this microstructure made of immobile and entangled dislocations.
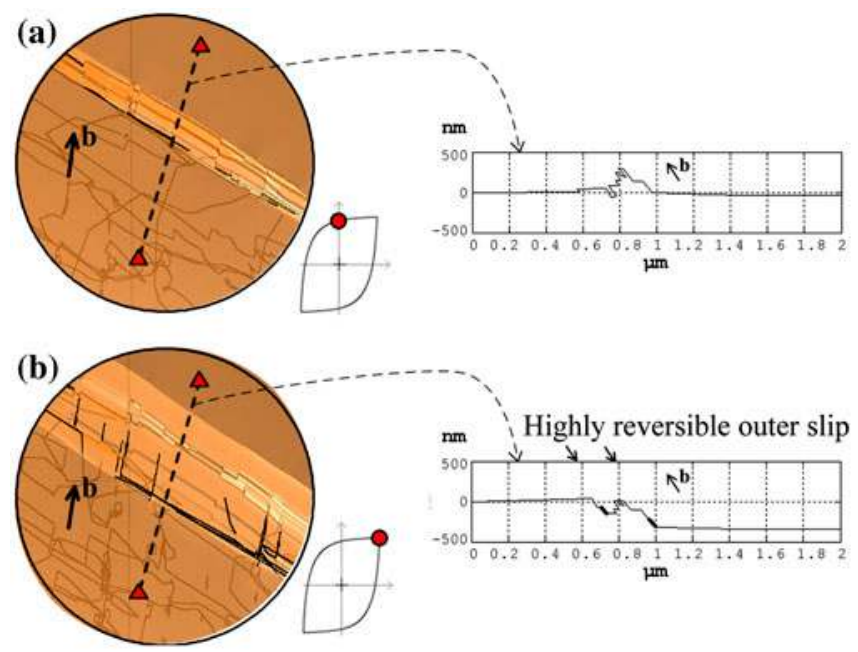

Figure 2. (colour online) Free surface relief at zero and maximum imposed strains during a single fatigue cycle. Highly reversible steps appear at the PSB-matrix interface, at maximum imposed strain. The surface displacements have been magnified $\times 50$, for clarity. 
(3) Mobile dislocations at the PSB-matrix interface. After few cycles, these dislocations accommodate most of the imposed plastic deformation. These mobile dislocations play a crucial role in extrusion growth. When the mobile dislocations glide in the PSB-matrix interface, they induce a stress gradient on multipoles nearby, which are subsequently swept towards the surface. On the opposite side of the grain, the mobile dislocations reaching the grain boundary create pileups, generating a back stress field that reduces the net stress acting on the inner PSB dislocation structures. Consequently, the dislocations located inside the PSB have a lower mobility and larger slip irreversibility. On the other hand, the mobile dislocations at the interface have a higher and higher slip reversibility which progressively leads to strong plastic strain localization at the PSB-matrix interface (see Figure 2). This situation yields to microcrack initiation at the interface and at the bottom of the extrusion, where the stress concentration is maximum [3-5]. In practice, the first crack will initiate when a set of multipoles, swept by the mobile interface dislocations, reach the free surface carrying the sufficient amount of energy needed for microcrack formation.

The aforementioned crack initiation mechanism applies to a broad range of imposed plastic strain amplitude. For plastic strain amplitude of $10^{-4}$ in $10 \mu \mathrm{m}$ grain at $20^{\circ} \mathrm{C}$, the typical band width is about $100 \mathrm{~nm}$. The band accommodates a maximum local plastic strain of about $10^{-2}$. As discussed later, the global dislocation density achieved in the grain at saturation is about $5 \times 10^{11} \mathrm{~m}^{-2}$ (see Figure 4(b)). In the bands, this corresponds to a local dislocation density of at least $5 \times 10^{13} \mathrm{~m}^{-2}$, mainly in the form of tangles and multipoles dislocations. In general, the simulated dislocation densities at saturation are lower than the experimental measurements. This is partly due to the simplifying assumptions of DD simulations, including the use of a discrete lattice and time step $\left(2 \times 10^{-10} \mathrm{~s}\right)$. The number of bands increases with the plastic strain amplitude, although the dislocation densities computed in individual bands remain constant. In any case, the number of dislocation gliding in the PSB-matrix interface and piling up at the bottom grain boundary is limited to $8-10$ dislocations. This number scales the size (depth) of the first microcrack developing from the surface (about $10 b=2 \mathrm{~nm}$ ), whereas the local shear stress near the grain boundary is about 10 times the applied stress.

\subsection{Stage-I fatigue crack propagation}

Section 3.1 and Figure 2 show that stage-I crack initiation and subsequent propagation most probably occurs where the slip is highly reversible, i.e. at the PSB-matrix interface, as reported in $[3,6]$. This is where a crack is now introduced in the simulations, as a combination of: (1) a free surface discontinuity (see Figure 3(a)), (2) a dislocation microstructure in front of the crack tip and (3) a heterogeneous stress field applied to the surrounding grain. When the grain size is small enough, it is possible to perform many cycles $(\sim 50)$, so that the crack is introduced when the saturation stage of the dislocation density is reached. For larger grains, CPU time limitation makes it more difficult to reach an important number of cycles $(\sim 8)$. Crack is then inserted during the transition stage.

The free surface discontinuity (1) associated with the crack is inserted in the form of a pair of intersecting free surfaces, with an opening angle of about $10^{-2}$ radians. 

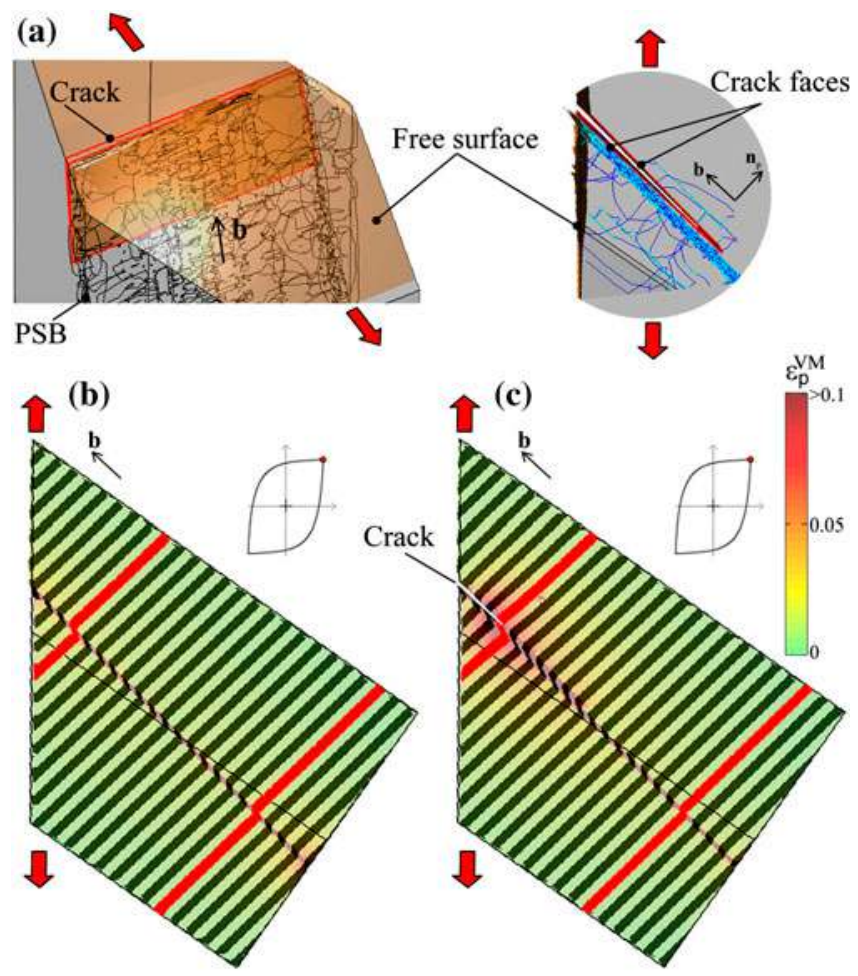

Figure 3. (colour online) (a) DD simulation geometry showing the introduction of crack at PSBmatrix interface; (b) slip displacement (represented by coloured strips) at maximum imposed strain in absence of crack, showing homogenous slip along the band. The background colour represents the intensity of the Von Mises plastic strain. (c) Slip displacements in the presence of a crack along the PSB-matrix interface, illustrating highly concentrated slip near the crack tip (compare red the strip patterns of Figure 3(b) and (c)).

These new surfaces act in the same way as the other grain free surfaces: outward motion of bulk dislocations is authorized, whereas any return is prohibited. As documented in [21], outcoming dislocations generate virtual dislocations that are only used to calculate the associated surface displacements, including a possible crack tip blunting effect. Elastic interactions between the virtual and bulk dislocations are switched off during the whole simulated time.

The additional dislocations (2) introduced with the crack consists of twelve additional FR sources, one per each possible FCC slip system. These sources are placed in front of the crack front and randomly distributed within $1 \mu \mathrm{m}$ from the crack front. Their role is to account for dislocation emission at the crack tip itself as evidenced experimentally. Different realizations of the initial configuration of the FR sources (number and positions) have been tested. The corresponding 3D simulations show that this has little effect on the subsequent microstructure and slip mechanisms following one complete (tension-compression) cycle. Indeed, each dislocation line emitted from the initial FR source easily cross-slip to a different slip plane leading to the formation 
of numerous new (Koehler-type) sources. For this reason, the number of sources is significantly increased after a few steps and the position of the initial artificial sources has no more effect.

Finally, the inserted crack induces in the grain a specific stress field (3). For the sake of computational speed and flexibility in changing the crack configuration (length, position and orientation), the crack stress field is introduced using Irwin's analytical expression [27]:

$$
\sigma_{i j}(r, \theta)=\frac{K_{\mathrm{I}}}{\sqrt{2 \pi r}} f_{i j}(\theta)+\frac{K_{\mathrm{II}}}{\sqrt{2 \pi r}} g_{i j}(\theta)+\frac{K_{\mathrm{III}}}{\sqrt{2 \pi r}} h_{i j}(\theta)
$$

$r$ and $\theta$ define the evaluation point coordinates. $K_{i}$ defines the mode- $i$ stress intensity factor ( $i=\mathrm{I}$, II or III). Equation (1) describes the stress field in front of an infinitely wide and sharp crack in a semi-infinite elastic continuum medium, i.e. it does not account for any blunting effect. It can be shown (using finite elements computations) that Equation (1) is a reasonably good approximation of the stress field acting in the grain, provided the ratio between the grain depth and the crack length is less than 5 . Another noteworthy point is that only $K_{\mathrm{I}}$ and $K_{\mathrm{II}}$ are non-zero factors in Equation (1), due to the specific orientation of the grain adopted in this study (uniaxial loading along the $\left[\begin{array}{lll}1 & 2 & 3\end{array}\right]$ direction). Consequently, crack plasticity potentially operates under tensile opening mode-I and/or in-plane sliding mode-II.

Figure 3(b) shows the slip distribution across the PSB before the crack is introduced. It can be seen that the cyclic slip is homogeneously distributed along the PSB length. Once the crack is introduced, the slip distribution is no longer homogeneous and becomes highly concentrated near the crack tip, along the primary slip plane coinciding with the stage-I crack plane (Figure 3(c)). In addition, the crack front geometry (observed at zero imposed strain during four successive cycles) shows very little evolution (Figure 4(a)), which evidences the reversibility of the slip activity at the crack tip. This effect is a direct consequence of the pre-existing PSB. In order to highlight that particular point, the same simulation is carried out with a crack introduced in an annealed grain, i.e. without any initial PSB microstructure. The outcome is then completely different (see Figure 4(d)): slip irreversibility near the crack tip is significant and leads to an increase in the crack length and significant changes in the crack shape, reminiscent of crack tip blunting effect. The obtained mechanism is comparable to that observed in crack growth under dominant mode-I (crack opening mode). In the 'PSBcrack' situation (case (a) in Figure 4), the dislocation substructure remains planar and no significant change is noticed except very near to the crack tip, where the dislocation density comes down as a consequence of enhanced slip reversibility. This simulated substructure is consistent with corresponding TEM and/or ECCI observations in fatigued FCC materials [5-8]. Experimental studies suggest that dislocation structures near a stage-I crack tip remain a PSB-ladder structure and the propagation of a short stage-I crack does not alter them significantly. In contrast, the dislocation substructure obtained in the second case (case (d) in Figure 4), where the crack is introduced from the beginning of the cycling, exhibits a thick tangled network around the crack tip.

Differences between the 'PSB-crack' and 'crack in an annealed grain' cases can also be quantified and partially explained based on the dislocation density evolutions on each slip system plotted for two different grain sizes in Figure 4(b)-(c) and (e)-(f), 

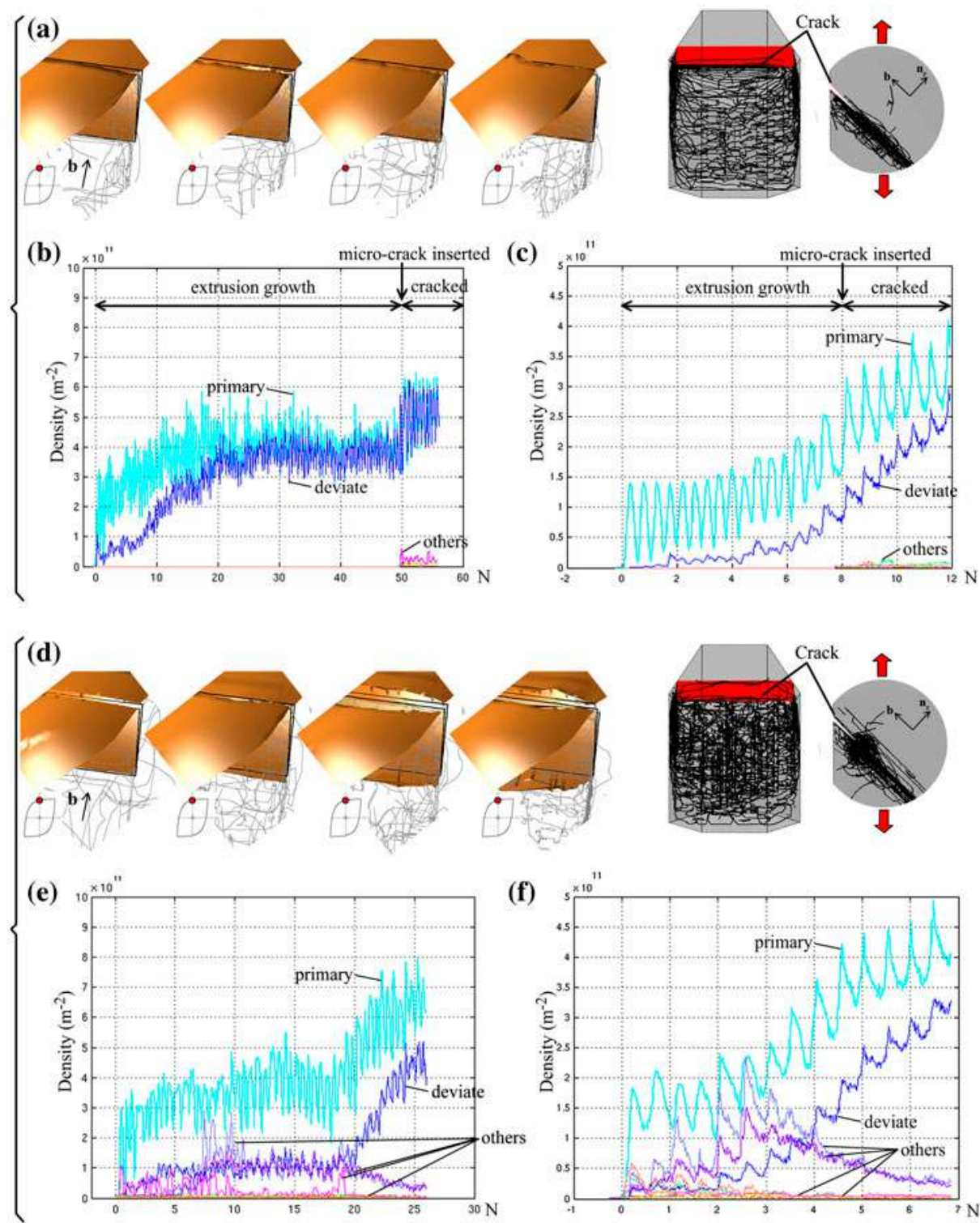

(e)

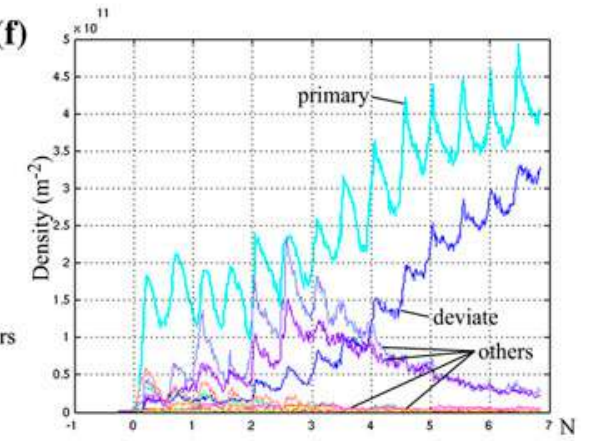

Figure 4. (colour online) (a) Plastic displacement evolutions at zero plastic strain, during five successive cycles. The introduction of a crack along the PSB ('PSB-crack' case) induces negligible changes on both the crack tip shape and the dislocation microstructure. Evolutions of the dislocation density on each slip system for (b) a $10 \mu \mathrm{m}$ grain and (c) a $20 \mu \mathrm{m}$ grain, in the 'PSBcrack' case. (d) Significant changes of the crack front shape and crack length are evidenced, when the crack is introduced without an initial PSB microstructure ('crack in an annealed grain' case). Dislocation density evolutions in each slip system for (e) a $10 \mu \mathrm{m}$ grain and (f) a $20 \mu \mathrm{m}$ grain, in the 'crack in an annealed grain' case. (g) Plastic displacement profiles through the free surfaces during one cycle (displacement magnification $\times 50$ ). 


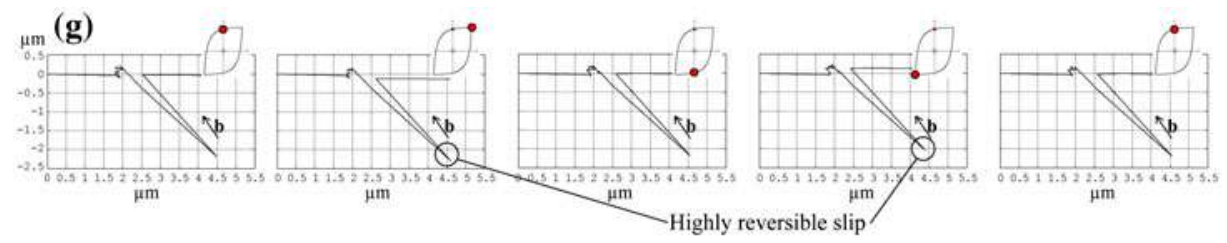

Figure 4. (Continued).

respectively. In the 'PSB-crack' case, Figure 4(b) and (c) reveals that before the crack is introduced, the dislocation density is mainly composed of dislocations gliding on the primary and the associated cross-slip systems, while the other slip systems remain inactive. The newly introduced crack has little effect on the dislocation density accumulation rate, which remains close to zero when the crack is introduced after saturation (Figure 4(b)) or follow the same evolution, when introduced during the transition stage (Figure 4(c)). This indicates that the plasticity around the crack tip fully operates with the existing PSB microstructure: no microstructural changes occur. The active plasticity mechanisms are the same as those described in Section 3.1: the imposed strain is mainly accommodated by mobile dislocations gliding at the PSB-matrix interface (and in front of the crack), whereas cross-slip activity along the band increases the density of existing tangles and multipoles. In the 'crack in an annealed grain' case, on the other hand, the primary and cross-slip systems are not the only preferentially active slip systems, since three more slip systems are also activated from the first cycles onwards (Figure 4(e) and (f)). Such a complex slip activity promotes the spreading of the dislocation substructure on a large volume surrounding the crack tip. This is consistent with the observed pronounced slip irreversibility and the corresponding crack growth mechanism. This is a genuine microstructural effect related to the number of active slip systems rather than a mere dislocation density effect, since the involved dislocations densities (in the main slip systems) are nearly the same, in either cases.

Figure 4(g) shows the crack front evolution during the PSB-crack simulation, over one complete fatigue cycle. Crack tip slip is found to be almost entirely reversible, which indicates that stage-I crack growth is compatible with successive decohesion events taking place across the crack plane along the PSB-matrix interface. In other words, stage-I crack growth is not merely associated to plastic irreversibility, as sometimes proposed in the literature. Stage-I crack growth mechanism is, thus, comparable to the microcrack initiation mechanism as described in Section 3.1. In practice, reversible crack tip slip (Figure 4(g)) favours the adsorption of non-metallic atoms (such as oxygen or hydrogen) in the tip region, by repeatedly exposing the same small surface to the environment. Adsorbed atoms can in turn reduce the decohesion threshold (through diminishing the interfacial surface energy), and therefore facilitates the subsequent stage-I crack propagation [28,29]. This decohesion process is similar to the wellknown hydrogen embrittlement mechanism observed under tensile loading conditions, where hydrogen accumulation at the crack front is favoured by notch geometries $[29,30]$ and drastically decreases the surface energy of the host metal [28]. 


\subsection{Crack propagation close to a grain boundary}

Stage-I crack propagation mechanism described in the previous section is somewhat modified, as the crack approaches the grain boundary. When the available grain space in front of the crack tip narrows down, plasticity developing in front of the crack (in the primary slip systems) is no longer sufficient to fully accommodate the imposed strain. Besides, slip dispersion near the crack front may be more or less pronounced, depending on the adjacent grain orientation. This means that grain orientations play an important role in the retardation/accentuation of stage-I crack propagation [31]. Understanding this mechanism is fundamental from the view point of crack propagation kinetics and the subsequent transmission/propagation to the adjacent grain. In the present study, plastic strain developed in the next grain is not taken into account, since the current version of the DD code can only handle a single crystal (or grain). However, crack propagation up to the first grain boundary can be treated with a full description of the dislocation activity in the cracked grain, providing valuable insight on grain boundary effects.

A crack is introduced in the form of two additional free surfaces along the PSBmatrix interface, i.e. using the same implementation method as described in the previous section. The crack front is now positioned at a pre-defined distance to the impenetrable grain boundary assuming that the initial propagation has occurred along the PSB interface, up to the current position. The next grain facing the crack front is then considered as a plastically undeformable elastic medium. Then, Equation (1) is not entirely accurate since the elastic anisotropy of each neighbouring grain is not taken into account and it may affect a small region near the grain boundary. The results are nevertheless consistent within the framework of isotropic elasticity used in the DD simulation.

Because of plastic constraints at the grain boundary, cyclic slip is no longer completely accommodated along the PSB-matrix interface (i.e. on primary slip system), but leads to the activation of additional slip systems ahead of the crack front (Figure 5(a)(c)). This effect results in the dispersion of the dislocation slip activity. Figure 5(a) clearly shows that plasticity expands on many planes parallel to the primary slip plane (by double cross-slip mechanisms), and also on secondary slip planes non-parallel to the crack plane. This configuration corresponds to complex crack tip morphology as shown in Figure 5(c), which could alter crack propagation kinetics and generate a crack tip stress field different from that of Equation (1), since this equation assumes an infinitely sharp crack tip configuration.

The effect of the grain size on the plastic strain spreading near the grain boundary is evidenced in Figure 5(a). Slip dispersion, indeed, decreases with the grain size, thus favouring slip localization. This means that grain-to-grain propagation should be easier for small grains, especially if the plastic deformation in the adjacent (non-cracked) grain is limited. This finding is consistent with experimental evidence [32] and is also highlighted through CTSD evaluation to be discussed in the next section. The severity of slip localization in front of the crack can be easily predicted using elementary physical considerations. When the crack is long enough and assuming that mode-II is preponderant, the mean shear stress acting in front of the crack is $\frac{1}{D g-a} \int_{0}^{D g-a} \frac{\tau_{a p p}^{\infty} \sqrt{\pi a}}{\sqrt{2 \pi r}} d r=\tau_{a p p}^{\infty} \sqrt{\frac{2 a}{D g-a}}$, where $a$ is the length of the crack. This stress generates a dislocation pile-up of $n$ dislocations in front of the crack, in turn producing a 
(a)

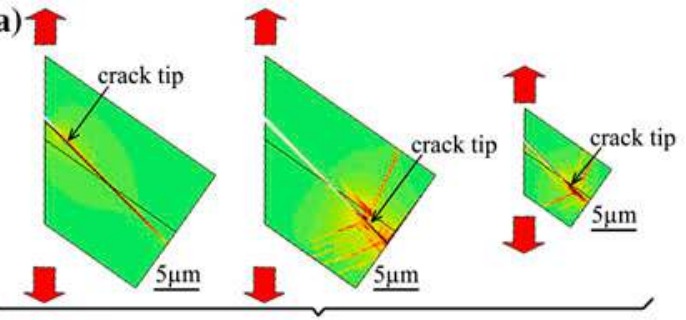

(b)

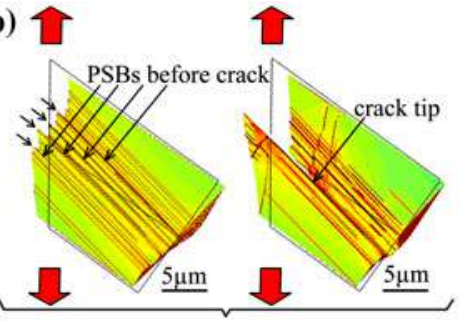

(c)
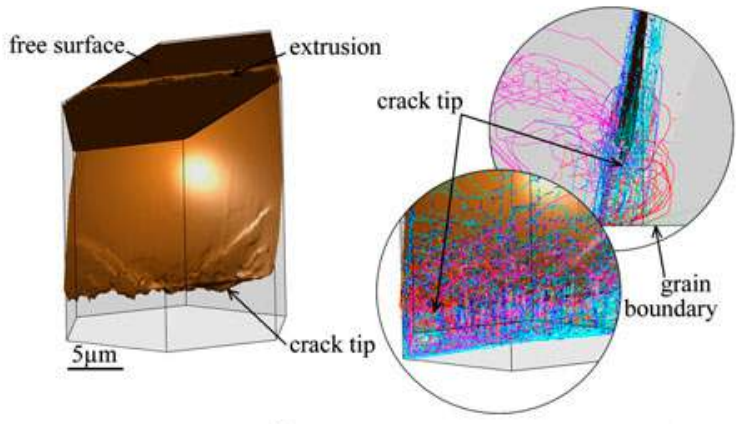

(d)
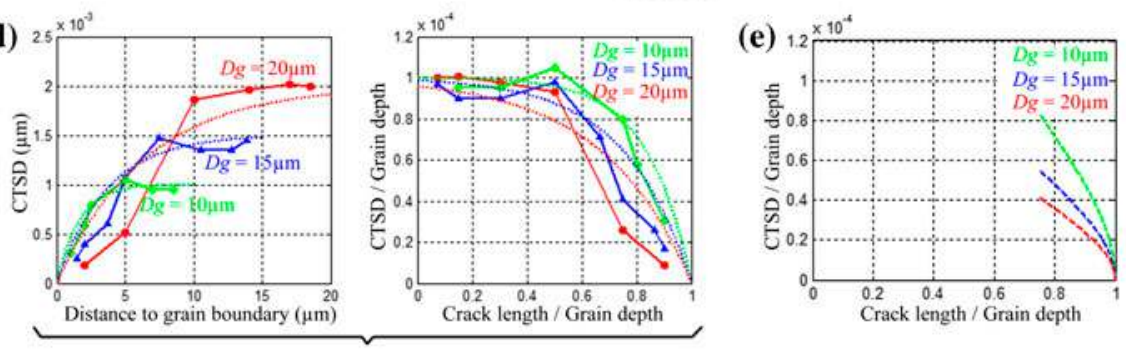

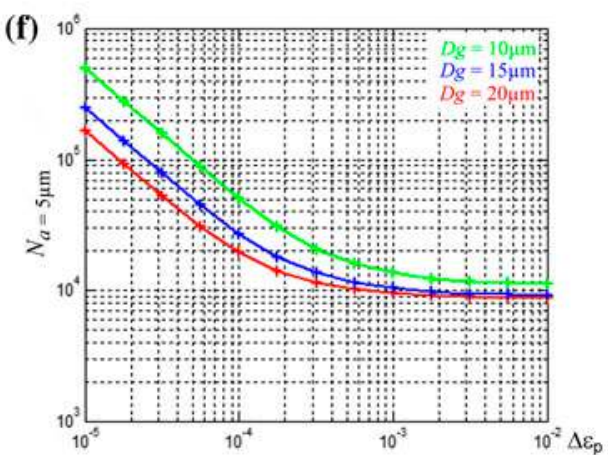

Figure 5. (colour online) (a) Von-Mises strain field for different crack front positions (from the grain boundary) and for 20 and $10 \mu \mathrm{m}$ grain depths. (b) Von-Mises strain field at higher imposed plastic strain $\left(5 \times 10^{-4}\right)$, before and after crack introduction. The displacement field is magnified $\times 30$ for clarity. (c) Crack front morphology and associated dislocation microstructure. (d) CTSD evolution as a function of the crack tip position for the three different grain depths, evaluated using Equation (3). (e) CTSD evolution as a function of crack tip position for the three tested grain depths, evaluated using Equation (2). (f) Number of cycle to get a $5 \mu \mathrm{m}$ length crack, evaluated using Equation (4). 
stress $n \times \tau_{a p p}^{\infty} \sqrt{\frac{2 a}{D g-a}}$ at the grain boundary. As mentioned in paragraph 3.1, the maximum stress acting in the grain boundary is about 10 times the applied stress $\tau_{a p p}^{\infty}$. This implies that the maximum number of dislocations which pile-up in front of the crack (before cross-slip activation) is:

$$
n=5 \sqrt{2} \sqrt{\frac{D g}{a}-1}=5 \sqrt{2} \sqrt{\frac{d_{\mathrm{gb}}}{D g-d_{\mathrm{gb}}}}
$$

where $d_{\mathrm{gb}}$ stands for the distance of the crack tip to the grain boundary. From Equation (2) it is obvious that, for a given distance $d_{\mathrm{gb}}$ to the grain boundary, the number of dislocations piling up in front of the crack actually decreases, with increasing the grain size.

For higher plastic strain amplitudes, numerous PSBs are present before crack initiation [20,21]. Many of these PSBs become inactive (Figure 5(b)) after crack initiation, because the crack tip tends to concentrate a large part of the imposed plastic strain amplitude. The small number $n$ of dislocations which can be emitted in front of the crack tip is then not sufficient to accommodate large plastic strain level. For this reason, slip dispersion is faster with increasing plastic strain amplitudes.

\subsection{Evaluation of the CTSD}

Previous observations are formalized in this section, by determining the CTSDs for different grain sizes and different positions of the crack front along the grain depth. In practice, the CTSD is computed by monitoring the displacements of control lines cutting through the crack and free surface of the grain, using the same calculation method as explained in [21]. The CTSD is evaluated for varying crack lengths $a$ in three selfsimilar grains of different depths, with a given 'crack-length/grain-depth $a / D g$ ' ratio. Figure 5(d) presents the evolution of the CTSD as a function of the crack length. It is obvious that the ratio $\mathrm{CTSD} / \mathrm{Dg}$ is almost independent on the grain size for crack length $a<D g / 2$. This means the CTSD is proportional to the grain depth so that the number of cycles needed for the crack to move over a distance $D g / 2$ is more or less the same, regardless of the grain size. The CTSD magnitude is affected by the grain depth as the crack approaches a grain boundary (see Figure 5(d)) owing to the previously explained slip dispersion effect. Namely, the CTSD magnitude reduction is more pronounced in coarse grains than in fine grains. Stage-I crack propagation is, therefore, slower in coarse grains, as reported in [27,33].

Under load, the dislocation sources in front of the crack tip generate dislocations that accommodate the imposed plastic strain and pile-up on the grain boundary, in front of the crack. One dislocation emitted from the crack tip can accommodate a maximum amount of plastic strain $\gamma_{\mathrm{p}}^{1}$, which is proportional to the maximum area $A$ swept before reaching the grain boundary, namely: $\gamma_{\mathrm{p}}^{1}=\frac{b . A}{V g}=\frac{b \cdot d_{\mathrm{gb}}}{D g^{2}}$, where length $d_{\mathrm{gb}}$ is the distance between the crack tip and the grain boundary and $b$ is Burgers vector magnitude. The

imposed plastic strain amplitude $\Delta \varepsilon_{\mathrm{p}}$ is fully accommodated if $n=\Delta \varepsilon_{\mathrm{p}} / \gamma_{\mathrm{p}}^{1}$ dislocations are emitted and pile-up in front of the crack. In practice, this condition is no longer satisfied when the distance to the grain boundary (measured from the crack front) becomes too small. This would, indeed, require a too large number of dislocations to accommo- 
date the imposed plastic strain on a single slip plane. Actually, cross-slip events driven by the internal stress would occur at the location of the dislocation pile-ups, spreading plasticity away from the crack tip region (Figure 5(a)). The implemented cross-slip mechanism, thus, contributes to reduce the CTSD (see Section 2). The stress difference $\tau_{\mathrm{III}}-\tau_{\mathrm{dev}}$ acting on the $n$th piled up dislocation is proportional to $1 / n$. The corresponding cross-slip probability is then proportional to $\exp \left(-\frac{\lambda}{n}\right)$. From the above considerations, the following expression is proposed to describe the CTSD evolution along the grain depth:

$$
\frac{\mathrm{CTSD}}{D g} \cong \Delta \varepsilon_{\mathrm{p}}\left(1-\exp \left(-\frac{\lambda}{\Delta \varepsilon_{\mathrm{p}}} \frac{b}{D g}\left(1-\frac{a}{D g}\right)\right)\right)=\Delta \varepsilon_{\mathrm{p}}\left(1-\exp \left(-\frac{\lambda}{\Delta \varepsilon_{\mathrm{p}}} \frac{b \cdot d_{\mathrm{gb}}}{D g^{2}}\right)\right)
$$

where $\lambda=25$ is a dimensionless fitting coefficient. This expression satisfactorily describes all the simulation data, as shown in Figure 5(d) (dashed curves). It is also worth noting that Equations (2) and (3) yield the same prediction for $a>D g / 2$. Indeed, the value of the dimensionless quantity $\mathrm{CTSD} / \mathrm{Dg}$ can be evaluated from Equation (2), assuming that $\mathrm{CTSD}=n b$. It gives $\frac{\mathrm{CTSD}}{D g}=5 \sqrt{2} \frac{b}{D g} \sqrt{\frac{D g}{a}-1}=5 \sqrt{2} \frac{b}{D g} \sqrt{\frac{d_{\mathrm{gb}}}{D g-d_{\mathrm{gb}}}}$, whose evolution is plotted in Figure 5(e) and found to be compatible with the data shown in Figure 5(d). This shows that the crack-induced strain spreading limits the stress acting in the grain boundary to about 10 times the applied stress.

The crack propagation rate can be quantified using Equation (3), assuming that a micro decohesion zone of size $\approx \mathrm{CTSD}$ forms at the crack tip every $N_{i}$ cycle. This gives:

$$
\frac{\mathrm{d} a}{\mathrm{~d} N}=\frac{\Delta \varepsilon_{\mathrm{p}}}{N_{i}}\left(1-\exp \left(-\frac{\lambda}{\Delta \varepsilon_{\mathrm{p}}} \frac{b}{D g}\left(1-\frac{a}{D g}\right)\right)\right)
$$

Integration of Equation (4) is given in Figure 5(f), indicating the number of cycle for a crack to reach a given length, for example, $5 \mu \mathrm{m}$ (noted $N_{a=5 \mu \mathrm{m}}$ ). At low imposed plastic strain amplitudes, $N_{a}=5 \mu \mathrm{m}$ is somewhat proportional to $\Delta \varepsilon_{\mathrm{p}}^{-1}$. At higher plastic strain amplitudes however, $N_{a}=5 \mu \mathrm{m}$ becomes more or less constant. This number of cycles is very low in comparison with the number of cycles to initiation, which was found to be proportional to $\Delta \varepsilon_{\mathrm{p}}^{-2}$ [21]. This clearly shows that initiation takes a much longer time than propagation, especially with decreasing $\Delta \varepsilon_{\mathrm{p}}$.

\subsection{Effect of crack opening/closure}

In the previous simulations, the effect of crack closure on the crack-induced stress field (during the compression phase) was not taken into account. In order to evaluate this effect, a separate simulation is run using a modified heterogeneous stress field. During the tension phase, the applied stress field is given by Equation (1), whereas during compression phase, mode-I contribution to Equation (1) is suppressed, to take into account the crack closure. Figure 6(a) and (b) shows the differences between the two situations, both in terms of shear stress on primary/deviate slip systems and applied macroscopic stress-plastic strain (SS) curve (resolved on primary slip system). Firstly, the crack introduction induces a slight softening of the grain in both cases, evidenced by the decrease of the overall stress amplitude. This softening is due to a decrease in the microstructure-induced back stress. When a short crack is introduced indeed, the resulting 
(a)
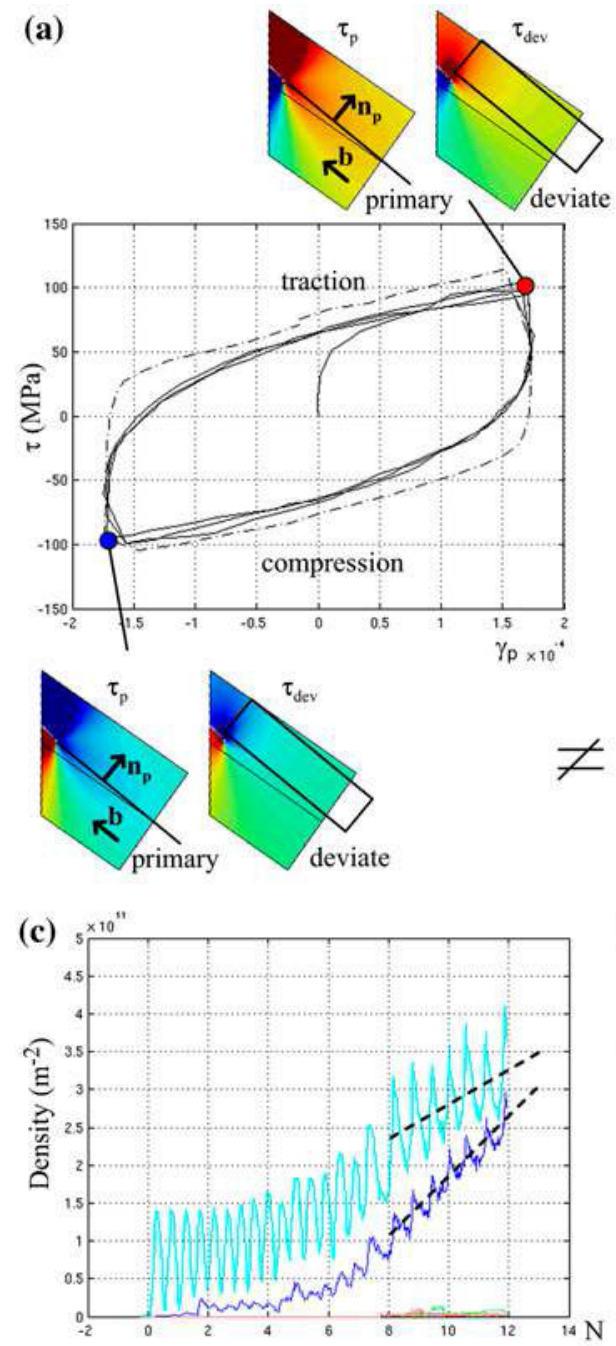

$\not$
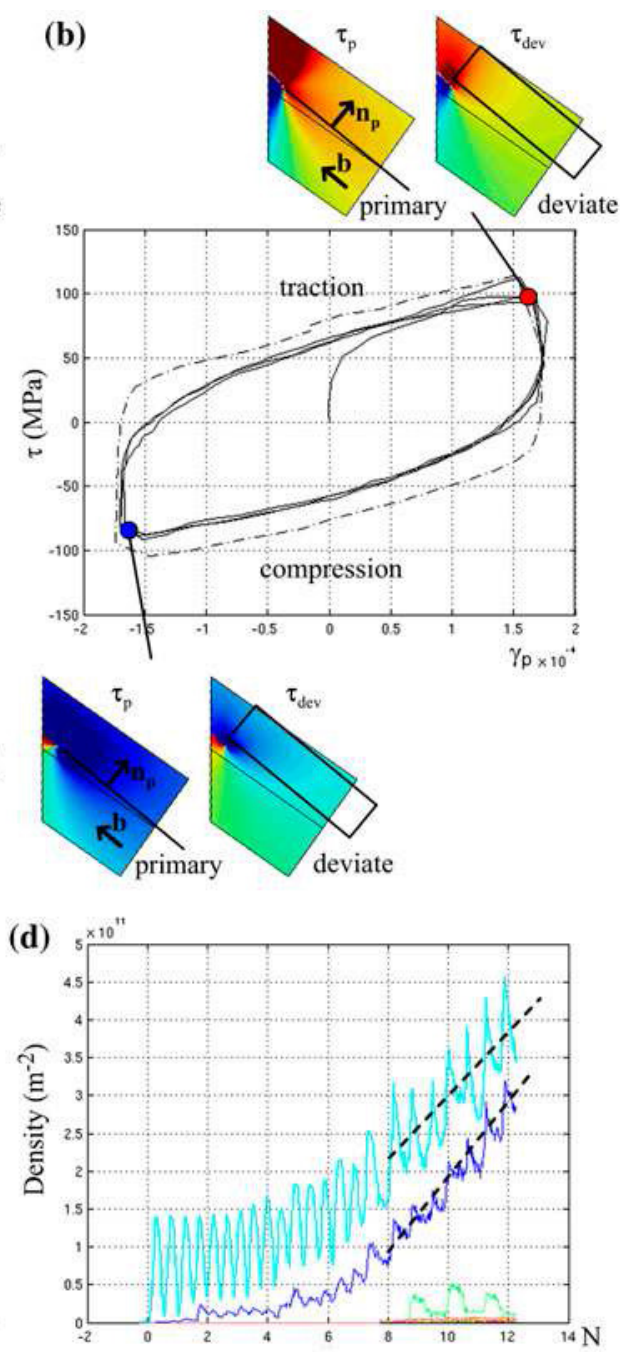

(e)

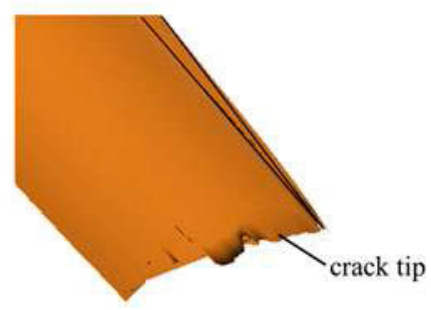

Figure 6. (colour online) (a) Stress vs. plastic strain evolutions (resolved on primary slip system) obtained without crack closure effect, and associated shear stress mapping resolved in primary and cross-slip systems. The dashed SS curves are obtained in the absence of crack. (b) The same as in (a) with active crack closure effect. (c) Dislocation density evolution without crack closure. (d) The same as in (c) with crack closure procedure activated. (e) Crack tip morphology after four cycles, with active crack closure effect, magnified $\times 100$. 
stress field favours a continuous distribution of the dislocations in front of the crack tip (see Figure 4(a)), which generates less back stress than dislocations piled up directly against the grain boundary (as it is the case in absence of a crack, see Figure 1). This softening (obtained here under constant applied strain amplitude) is fully comparable to the cyclic creep observed in a very recent DD study [15], in which the crack is loaded under constant applied stress amplitude. Secondly, the macroscopic effect of crack closure results in a slight dissymmetry of the SS curve (the overall amplitude is slightly decreased, whereas a small positive mean stress is generated). Once again, this is due to the distribution of the stress in front of the crack, which is more homogeneous in the absence of mode-I loading component (compare the stress mapping in compression with and without crack opening). This situation further homogenizes the dislocation spreading in front of the crack and hence, further decreases the back stress. In terms of dislocation density, a marked effect of the crack closure is noted (compare Figure 6(c) and (d)). The increase in dislocation accumulation rate, already evidenced in paragraph 3.3 and in Figure 4 when crack closure effect is inactive, is significantly enhanced in both the primary and cross-slip systems, when the crack closure procedure is active. This effect can be explained in terms of irreversible dislocation slip, associated with the dissymmetric tension and compression phases of the cycle. Moreover, the increased shear stress on the cross-slip system induced by the crack closure tends to increase the probability of glide path change. As a result, the dislocation microstructure generated near the crack tip is slightly thicker and denser than before. This induces markings in the crack tip surfaces (Figure 6(e)), attesting of a high level of slip irreversibility. Overall, the introduction of crack closure decreases the CTSD and slows down the crack growth rate. These effects are of the second order, however, and for this reason, the analysis and conclusions of the previous sections need no revision.

\subsection{Most favourable crack position along a PSB}

It is well known that cracks initiate in the vicinity of pre-existing PSBs. However, the factors controlling the actual microcrack growth path along the bands are still unclear $[3,6]$. Two additional DD simulations have been conducted, with a view to compare the crack growth behaviour depending on its position, in presence of a pre-existing PSB: a

(a)

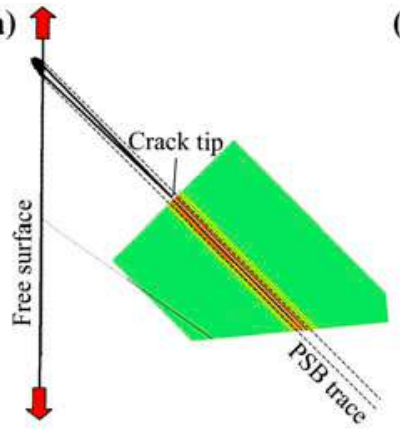

(b)

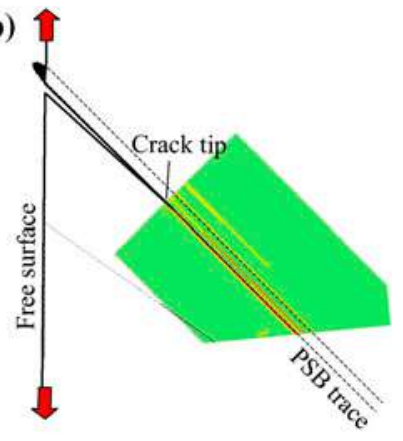

Figure 7. (colour online) Plastic strain mapping in front of the crack tip. (a) Crack introduced in the PSB centre. (b) Crack introduced in the left-sided PSB-matrix interface. 
simulation where the crack is introduced in the PSB centre and a simulation where the crack is introduced in the left PSB-matrix interface. It can be seen in Figure 7 that the two tested positions are not equivalent. When the crack is introduced at the PSB centre, band thickening, enhanced slip irreversibility crack effects are noticed. The PSB microstructure spreads out ahead of the crack and includes many additional (out of band) slip planes, a situation that is very ineffective to accommodate the locally imposed plastic strain. Because of enhanced slip spreading, the crack tip is no longer sharp, whereas the CTSD is significantly decreased. The centre crack position (or path) is, therefore, unfavourable for propagation along the PSB (see also paragraph 3.1). If we now introduce the crack along the left PSB-matrix interface, we can see that plasticity around the crack tip remains as sharp as before. The CTSD is, however, smaller in the left-sided than in the right-sided crack case, due to the lower stress concentration associated with the former case [3-5], as reported in paragraph 3.1. This means that the left-side position is unfavourable for subsequent crack propagation, at least when the crack is short enough.

\subsection{Influence of the image forces}

All the simulations presented so far in this paper have neglected the influence of image forces associated to the free surfaces (though the geometrical crack singularity effect is included). This choice was made with a view to minimize the computational load. Neglecting the image forces means that the traction-free condition $T_{\mathrm{i}}=\sigma_{\mathrm{ij}} n_{\mathrm{j}}=0$ is not verified in the free surfaces of the simulated volume, in the presence of combined applied and internal stress fields. Previous studies have shown that this approximation

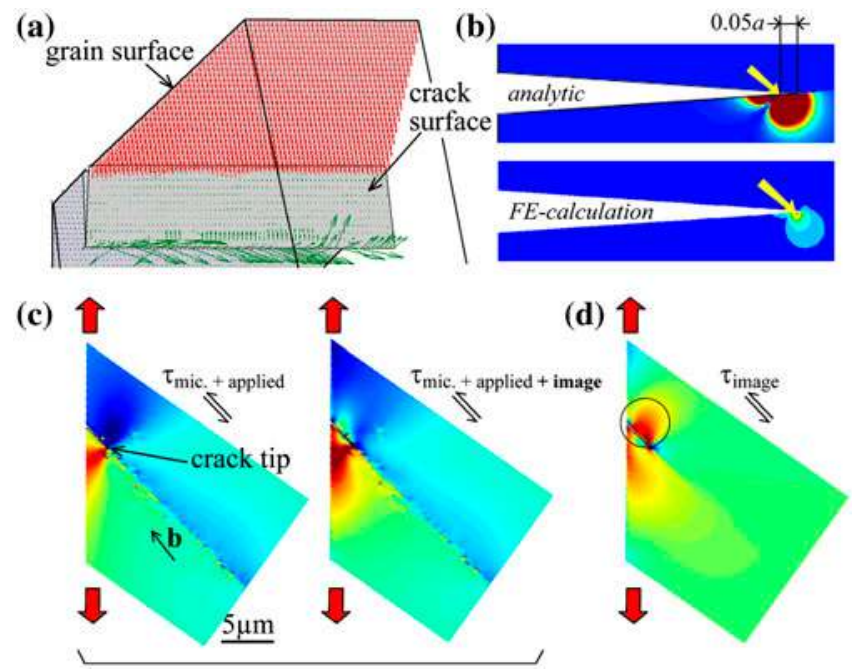

Figure 8. (colour online) (a) Traction vector acting in the different free surfaces. (b) Von Mises stress field evaluated using analytical (using point forces [34]) and FE calculation solutions. Good accuracy of the analytical solution, including in the vicinity of the crack tip, can be noticed. (c) Stress field (shear component on the primary slip plane) calculated without and with image forces. (d) Image stress field acting in the simulated grain. 
has little effect on PSB arrangements and their evolutions, at least up to microcrack initiation [21]. In the case of a crack at the PSB-matrix interface, however, additional free surfaces are positioned close to the PSB and for this reason, image forces may have a greater effect than before. The surface traction vector field $T_{\mathrm{i}}$ is sketched in Figure 8(a) at the maximum imposed strain. As expected, the traction-free surface condition is not satisfied $\left(T_{\mathrm{i}} \neq 0\right)$. The image stress field is evaluated at the same calculation step, using the analytical method described in [34]. This method consists in applying a field of point forces $F_{\mathrm{i}}$ on the grain-free surfaces, which opposes to the surface traction vector field: $F_{\mathrm{i}}=-T_{\mathrm{i}}$. The stress field induced by each point force is then calculated using the analytical solution of Boussinesq [34]. Comparison with FE calculations show that Boussinesq method provides a good estimate of the image stress everywhere in the simulated volume (see Figure $8(\mathrm{~b})$ ). The stress fields obtained with and without the image forces are then compared side by side in Figure 8(c), where the shear stress component along the primary slip plane is represented at the maximum plastic strain. Firstly, we notice that the difference between the two cases is quite small. Secondly, the shear stress along the notch surfaces nearly vanishes in the presence of the image field, thus validating the selected image field calculation method. Moreover, the image field range is restricted to a circular area circumscribed to the crack, as evidenced in Figure 8(d). This observation argues in favour of a limited influence of the image field on the slip in front of the crack.

The image field influence across the whole grain is now evaluated by comparing the average elastic energy per unit of volume $E=\frac{1}{\Omega} \int_{\Omega} \frac{1}{2} \sigma: \varepsilon_{e} \mathrm{~d} \Omega$ stored in the grain, with and without images field. The stored energy in the absence of image forces is about $67.10^{-3} \mathrm{~J} \mathrm{~m}^{-3}$ vs. $71.10^{-3} \mathrm{~J} \mathrm{~m}^{-3}$, in the presence of image forces. This small difference $(\sim 6 \%)$ confirm the limited influence of the image field in DD simulations conducted at the grain size scale, even in presence of a crack-like discontinuity.

\subsection{Energy balance during the crack propagation}

The CTSD calculations presented in the previous section suggest a possible stage-I crack propagation mechanism. Another way to envisage crack propagation is to study the energy changes within the grain, for a given crack advance. In DD simulations, the local elastic strain energy per unit of volume is computed as $e=K_{\mathrm{ijkl}}^{-1} \sigma_{\mathrm{ij}} \sigma_{\mathrm{kl}}$, where $K$ and $\sigma$ are, respectively, the Hooke tensor and the local effective stress tensor. The latter quantity accounts both for the applied heterogeneous stress due to the crack and the internal stresses induced by the dislocation microstructure. The image field is not taken into account here, since it represents only a little fraction of the total stored energy (see Section 3.7). Figure 9(a) shows a typical mapping of the applied elastic energy induced by the crack only computed in the entire grain. Figure 9 (b) shows the total elastic strain energy taking into account both the crack and the dislocation stresses. Comparison of Figure 9(a) and (b) highlights the stress relaxation effect induced by the dislocation microstructure.

The effect of the crack propagation on the elastic strain energy stored in the material is now estimated for a stabilized dislocation microstructure submitted to a given constant stress. In practice, one DD simulation including a crack along PSB-matrix interface is first carried out during a few cycles. Then, the microstructure is stabilized at the peak tensile stress until the plastic strain rate vanishes, in order to get a fully 

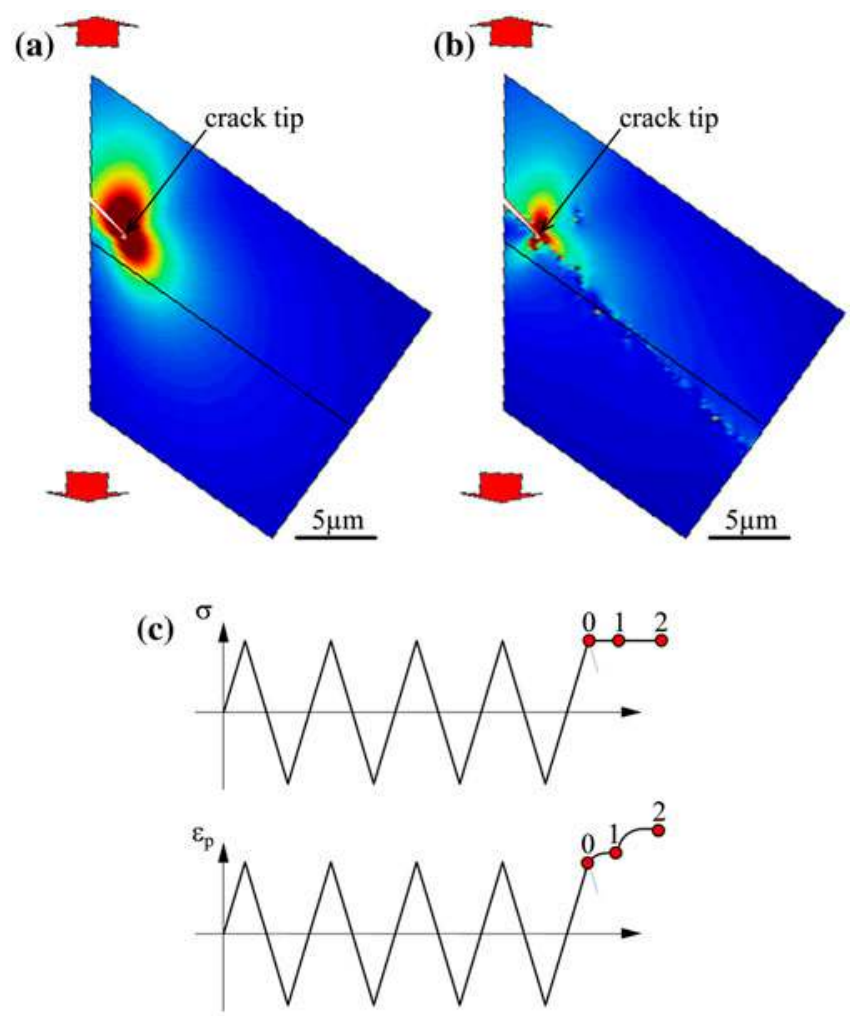

Figure 9. (colour online) (a) Applied elastic strain energy field due to the crack. (b) Total elastic energy showing relaxation induced by the dislocation microstructure. (c) Schematic sketch of the applied stress and the resulting plastic strain showing the microstructure stabilization phase $(0-1)$ and destabilization-stabilization phase induced by a small advance of the crack (1-2).

equilibrated dislocation microstructure. This phase is shown as $0-1$ in Figure 9(c). After stabilization, the stored elastic strain energy $\left(E_{1}\right)$ is calculated. The crack front is then spontaneously extended for a small length $\mathrm{d} a$, whereas the applied stress remains fixed at the same value. The applied heterogeneous stress field is consequently slightly modified, which induces a small destabilization of the dislocation microstructure associated with a plastic strain burst. This phase is denoted as 1-2 in Figure 9(c). The dislocation microstructure is again stabilized and the elastic strain energy $\left(E_{2}\right)$ after the crack increment is calculated. This procedure has been applied for three different crack advances $\mathrm{d} a=10,30$ and $100 \mathrm{~nm}$, and the changes in the plastic strain and the associated dislocation density are shown in Figure 10(a) and (b), respectively.

Theoretically, it is assumed that the crack will propagate provided the elastic energy change induced by the crack length increment satisfies the fundamental principles of thermodynamics, which in the case of a crack advance under the constant applied stress, $\Sigma_{\text {app }}$, can be formulated as $[27,35]$ : 

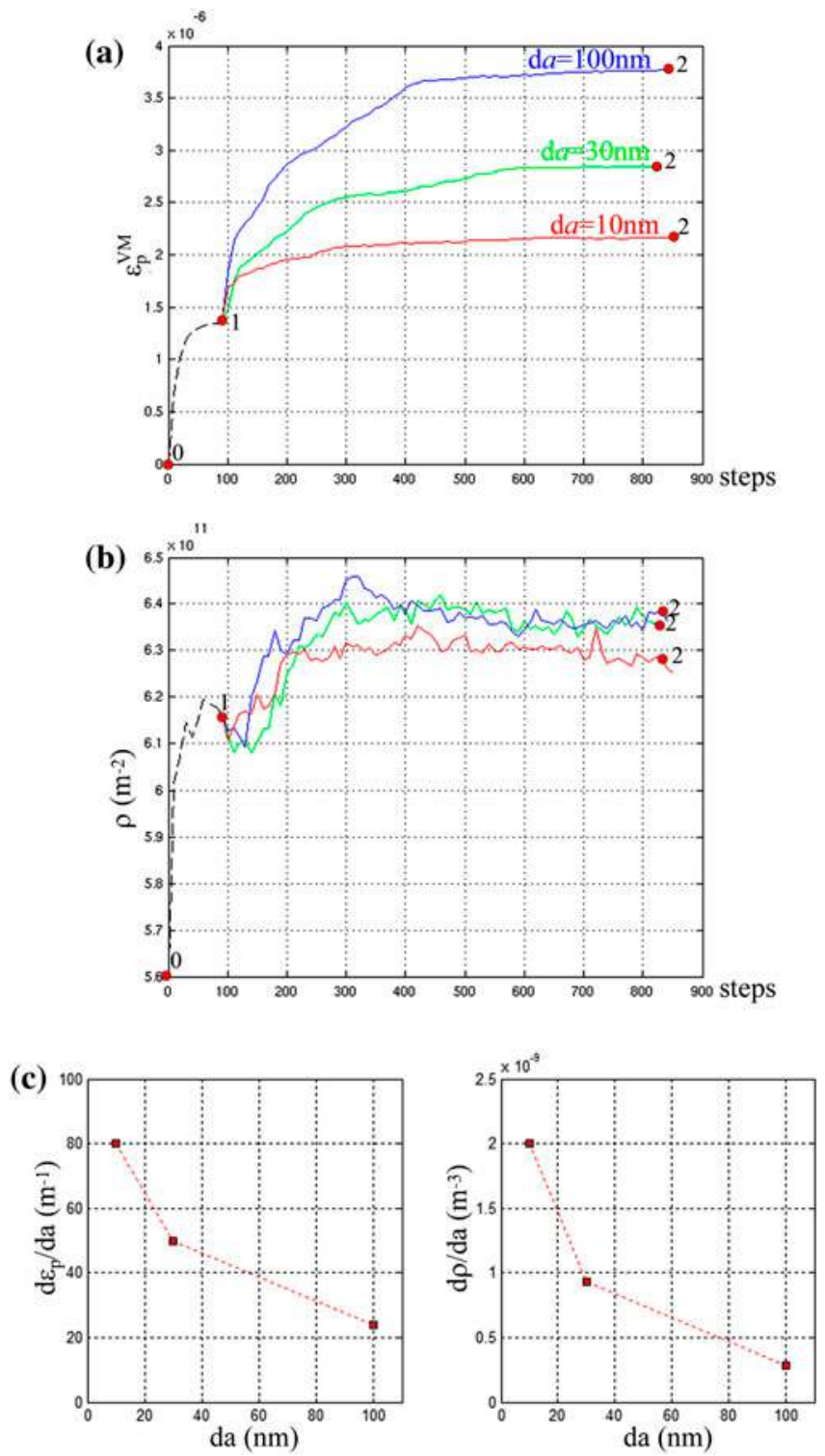

Figure 10. (colour online) Effect of spontaneous crack advance $\mathrm{d} a$ at constant stress on variations of (a) Von Mises plastic strain; (b) dislocation density, as a function of the number of time steps; (c) variation of $\mathrm{d} \varepsilon_{\mathrm{p}} / \mathrm{d} a$ and $\mathrm{d} \rho / \mathrm{d} a$ induced by a given crack advance $\mathrm{d} a$.

$$
G_{c} \mathrm{~d} A \leq \mathrm{d}\left(\int_{\Omega} \frac{1}{2} \sigma: \varepsilon_{e} \mathrm{~d} \Omega\right)+\Sigma_{\text {app }} d \varepsilon_{\mathrm{p}} \Omega
$$

where the left-hand term $G_{c} \mathrm{~d} A$ is the surface energy change induced by the creation of additional free surface $\mathrm{d} A$ due to the propagation $(\mathrm{d} A=l \mathrm{~d} a$, where $l$ is the crack width). 
Table 2. Energy changes during early stage-I crack propagation for a given crack advance at a constant stress.

\begin{tabular}{|c|c|c|c|}
\hline$G_{\mathrm{c}} \mathrm{d} A\left(\times 10^{-13} \mathrm{~J}\right)$ & $\begin{array}{c}10 \\
2\end{array}$ & $\begin{array}{c}30 \\
6\end{array}$ & $\begin{array}{c}100 \\
20\end{array}$ \\
\hline $\mathrm{d}\left(\int_{\Omega} \frac{1}{2} \sigma: \varepsilon_{e} \mathrm{~d} \Omega\right)=E_{2}-E_{1}\left(\times 10^{-16} \mathrm{~J}\right)$ & 0.0016 & 0.0048 & 0.46 \\
\hline $\begin{array}{l}\Sigma_{\text {app }} \mathrm{d} \varepsilon_{\mathrm{p}} \Omega\left(\times 10^{-16} \mathrm{~J}\right) \\
\text { Propagation scenario }\end{array}$ & $\begin{array}{c}1.11 \\
\text { Unfavorable }\end{array}$ & $\begin{array}{c}1.86 \\
\text { Unfavorable }\end{array}$ & $\begin{array}{c}2.98 \\
\text { Unfavorable }\end{array}$ \\
\hline
\end{tabular}

The middle term is the change in elastic strain energy induced by the change in crack length and in the associated dislocation microstructure evolution. The last term (in third position in Equation (5)) is the dissipated energy due to plastic slip, i.e. dislocation motion, while $\Omega$ is the grain volume. The left-hand term can be calculated assuming a typical surface energy density $G_{c}=1 \mathrm{~J} . \mathrm{m}^{-2}$. The middle term is calculated from energy differences $E_{2}-E_{1}$ between two different, stabilized configurations. The right-hand term is directly calculated from Figure 10(a), which provides the plastic strain variation $\mathrm{d} \varepsilon_{\mathrm{p}}$ between the same two configurations. The different terms corresponding to the three tested crack advances are tabulated in Table 2.

It is shown that the crack surface energy required for the selected crack advances is much higher than the sum of the elastic and plastic energy changes. It means that spontaneous crack propagation is not favourable for the above-tested crack advances, at the selected constant stress levels. However, two noteworthy points must be mentioned. Firstly, the actual surface energy may be decreased by several orders of magnitude if detrimental foreign atoms are adsorbed at the crack tip surfaces during the cyclic slip. As described in Figures 3 and 4, the pronounced plastic slip localization in the slip plane crossing the crack tip favours the contamination of the crack tip zone by nonmetallic atoms. Secondly, the crack advances tested in this study are quite large compared with the CTSD values given in Figure 5, suggesting smaller crack advances: for example, about $2 \mathrm{~nm}$ for $\Delta \varepsilon_{p}=10^{-4}$ and $D g=20 \mu \mathrm{m}$.

Elastic and plastic energy changes due to crack increments lower than $10 \mathrm{~nm}$ are about the same magnitude as the noise induced by random dislocation motion. This noise is due to the explicit temporal integration of the DD scheme. For this reason, effects of very small crack length increments (less than $10 \mathrm{~nm}$ ) cannot be investigated using the above-described method. However, it is possible to estimate a crack length increment compatible with the actual crack propagation by comparing the dislocation density derivative $\mathrm{d} \rho / \mathrm{d} a$ and the plastic strain derivative $\mathrm{d} \varepsilon_{\mathrm{p}} / \mathrm{d} a$ evolutions as a function of the crack length increment $\mathrm{d} a$ (see Figure 10(a)-(b)). And hence, Equation (5) can be reformulated, by considering that a part of the elastic strain energy change is induced by the evolution of the dislocation microstructure. As seen in Figure 10(b), a crack advance induces a small increase in the total dislocation density. The elastic energy change associated to a density variation $\mathrm{d} \rho$ is approximately $\beta \mu b^{2} \mathrm{~d} \rho$. In this equation, $\mu$ is the elastic shear modulus, $b$ is the Burgers vector amplitude and $\beta$ is a dimensionless coefficient, depending on the dislocation arrangement considered. For a small crack advance, $\beta$ can be considered as constant, since the microstructure is not drastically modified. Consequently, Equation (5) can be expressed as: 


$$
G_{c} \leq \beta \mu b^{2} \frac{\mathrm{d} \rho}{\mathrm{d} A}+\ldots+\Sigma_{\text {app }} \frac{\mathrm{d} \varepsilon_{\mathrm{p}}}{\mathrm{d} A} \Omega
$$

where the suspension points denote the elastic strain energy changes induced by the increase of the crack length, in a pure elastic medium. Increase of the derivatives in the two terms of Equation (6) should promote crack propagation. Derivatives $\mathrm{d} \rho / \mathrm{d} a$ and $\mathrm{d} \varepsilon_{\mathrm{p}} / \mathrm{d} a$ are plotted in Figure 10(c). Extrapolation of these curves shows that only small crack advances will induce important energy change (in Equation (6)). This clearly shows that crack propagation is highly favourable for very small crack advances, possibly $<<10 \mathrm{~nm}$, as seen in Figure 10(c).

\section{Summary}

The main results of this extensive 3D DD investigation of stage-I fatigue crack propagation are as follows.

- The crack-induced stress field yields a considerable reduction in the dislocation density near the crack tip during stage-I crack growth along the PSB-matrix interface. The resulting planar dislocation microstructure promotes highly reversible crack tip slip, without significantly altering the crack length and shape. This confirms that early stage-I crack propagation does not significantly change the prior PSB dislocation microstructure and proceeds through a progressive decohesion of the crystallographic planes along the PSB-crack interface.

- In the absence of prior-PSB microstructure, crack tip-induced plastic slip spreads into different slip systems. The associated slip dispersion increases the slip irreversibility, changes the crack tip morphology and the associated propagation mechanism.

- For a crack length $a<D g / 2$, the CTSD/Dg ratio is almost independent of the grain size. The CTSD magnitude is affected by the grain depth as the crack approaches a grain boundary, because of the slip dispersion effect. Consequently, the CTSD magnitude reduction is more pronounced in large grains than in small grains.

- The image forces induced by the presence of the crack surfaces have very little impact on stage-I crack propagation. The change in the elastic energy stored in the simulated volume is less than $10 \%$, which confirms that image forces can be neglected in DD simulations of fatigue crack growth.

- Only very small crack extension triggers a significant increase in the evolution of the dislocation density and the corresponding plastic strain, providing favourable conditions for early stage-I crack growth. This suggests that crack propagates by very small increments, most probably smaller than $10 \mathrm{~nm}$.

\section{Acknowledgements}

The authors acknowledge the financial support of French National Agency for Research through program AFGRAP ANR-08-MAPR-0024-05. The authors also thank Dr Baldev Raj, Former Director IGCAR, Kalpakkam, and Dr S.C Chetal, Director IGCAR, Kalpakkam, for their kind encouragement. 


\section{References}

[1] T. Kruml, J. Polak, K. Obrtlik and S. Degallaix, Acta Mater. 45 (1997) p.5145.

[2] C. Blochwitz, J. Polak, J. Man and K. Obrtlik, Acta Mater. 50 (2002) p.3767.

[3] J. Man, T. Vystavěl, A. Weidner, I. Kuběna, M. Petrenec, T. Kruml and J. Polák, Int. J. Fatigue 39 (2012) p.44.

[4] B.T. Ma and C. Laird, Acta Metall. 37 (1989) p.325.

[5] J. Ahmed, A.J. Wilkinson and S.G. Roberts, Phil. Mag. A 81 (2001) p.1473.

[6] K. Katagiri, A. Omura, K. Koyanagi, J. Awatani, T. Shiraishi and H. Kaneshiro, Metall. Trans. A 8 (1977) p.1769.

[7] J. Ahmed, A.J. Wilkinson and S.G. Roberts, J. Microscopy 195 (1999) p.197.

[8] K. Yoshihisa, I. Masao and H. Satoshi, Mater. Sci. Eng. 400 (2005) p.418.

[9] B.A. Bilby, A.H. Cottrell and K.H. Swinden, Proc. Roy. Soc. Lond. 272 (1963) p.304.

[10] R.W. Lardner, Proc. Roy. Soc. Lond. 317 (1970) p.199.

[11] E.R. De los Rios, Phil. Mag. A 57 (1988) p.15.

[12] A.J. Wilkinson, S.G. Roberts and P.B. Hirsch, Acta Mater. 46 (1998) p.379.

[13] V. Doquet, Fat. Fract. Eng. Mater. Struct. 21 (1998) p.661.

[14] V.S. Deshpande, A. Needleman and E. Van der Giessen, Acta Mater. 51 (2003) p.1.

[15] M. Huang, J. Tong and Z. Li, Int. J. Plasticity 54 (2014) p.229.

[16] B. Künkler, O. Düber, P. Köster, U. Krupp, C.P. Fritzen and H.J. Christ, Eng. Fract. Mech. 75 (2008) p.715.

[17] P. Hansson and S. Melin, Eng. Fract. Mech. 75 (2008) p.1400.

[18] F.O. Riemelmoser, R. Pippan and H.P. Stüwe, Acta Mater. 46 (1998) p.1793.

[19] C. Bjerkén and S. Melin, Int. J. Solids Struct. 46 (2009) p.1196.

[20] C. Déprés, C.F. Robertson and M.C. Fivel, Phil. Mag. 84 (2004) p.2257.

[21] C. Déprés, C.F. Robertson and M.C. Fivel, Phil. Mag. 86 (2006) p.79.

[22] M. Fivel and C. Déprés, Phil. Mag. 92 (2014) p.3206.

[23] M. Verdier, M. Fivel and I. Groma, Model. Simul. Mater. Sci. Eng. 6 (1998) p.755.

[24] L. Tabourot, M. Fivel and E. Rauch, Mater. Sci. Eng. 234 (1997) p.639.

[25] M. Fivel, C. Robertson, G. Canova and L. Boulanger, Acta Mater. 46 (1998) p.6183.

[26] C. Déprés, M. Fivel and L. Tabourot, Scripta Mat. 58 (2008) p.1086.

[27] S. Suresh, Fatigue of Materials, Cambridge University Press, Cambridge, 1998.

[28] D.E. Jiang and A. Carter, Acta Mater. 52 (2004) p.4801.

[29] C. Ayas, V.S. Deshpande and N.A. Fleck, J. Mech. Phys. Solids 63 (2014) p.80.

[30] P. Novak, R. Yuan, B.P. Somerday, P. Sofronis and R.O. Ritchie, J. Mech. Phys. Solids 58 (2010) p.206.

[31] G.V. Prasad Reddy, C. Robertson, C. Déprés and M. Fivel, Acta Mater. 61 (2013) p.5300.

[32] A. Van der Ven and G. Ceder, Acta Mater. 52 (2004) p.1223.

[33] C. Blankenship and E. Starke, Fat. Frac. Eng. Mat. Str. 14 (1991) p.103.

[34] M. Fivel, T. Gosling and G. Canova, Modelling Simul. Mater. Sci. Eng. 4 (1996) p.586.

[35] J.R. Rice, Int. J. Fract. Mech. 3 (1967) p.19. 